\title{
1 miR-92a-3p controls cell cycle progression in zebrafish
}

2 Running title: miR-92a-3p controls the cell cycle

\author{
Christopher E. Presslauer ${ }^{1}$, Teshome T. Bizuayehu ${ }^{1,2}$, Jorge M.O.
}

Fernandes ${ }^{1}$, Igor S. Babiak ${ }^{1 *}$

${ }^{1}$ Genomics Group, Faculty of Biosciences and Aquaculture, Nord University, 8049 Bodø, Norway

${ }^{2}$ Current address: Computational biology unit, Institute of informatics; Sars

*Corresponding Author: Igor Babiak. Faculty of Biosciences and Aquaculture, Nord

13 University, 8049 Bodø, Norway. Tel: +47 75517922

14 E-mail: igor.s.babiak@nord.no

16 Keywords: cell cycle, embryogenesis, microRNA, miR-92a-3p, wee2, zebrafish 


\section{Summary statement}

18

19 In zebrafish, maternal miR-92a-3p was demonstrated to suppress translation of wee2,

20 a cyclin-dependent kinase 1 inhibitor which regulates cell cycle progression during the

21 early stages of embryogenesis. 


\section{Abstract}

Biological functions of micro RNAs (miRNAs) in the early stages of vertebrate

25 development remain largely unknown. In zebrafish, miRNA miR-92a-3p is abundant in

26 the germ cells throughout gonadal development, as well as in ovulated oocytes.

27 Previously, we demonstrated that inhibition of miR-92a-3p in mature ovaries resulted

28 in developmental arrest at the 1-cell stage upon fertilization of the affected oocytes.

29 This suggested functions of miR-92a-3p in early development. In the present study, we

30 identified wee2, an oocyte-specific protein tyrosine kinase, as a target of maternal

31 miR-92a-3p during the early stages of zebrafish embryogenesis. Spatiotemporal co-

32 presence of both miR-92a-3p and wee2 during early embryo development was

33 confirmed by absolute quantification and in situ hybridization. Targeted knockdown of

34 miR-92a-3p in embryos resulted in retarded embryonic development over the first 24

35 hours. Target validation assays demonstrated that miR-92a-3p interacted with the

36 predicted wee2 3'UTR binding site, which was strongly suppressed by endogenous

$37 \mathrm{miR}-92 \mathrm{a}-3 \mathrm{p}$. Our results suggest that $\mathrm{miR}-92 \mathrm{a}-3 \mathrm{p}$ regulates the abundance of wee2, a

38 cyclin-dependent kinase 1 inhibitor, thus having important role in regulation of the cell

39 cycle during cleavage stages in zebrafish. 
40

41

42

43

\section{Introduction}

MicroRNAs (miRNAs) are a family of small non-coding RNAs which function in post-transcriptional repression of protein-coding genes (Bartel 2009). A miRNA along with Argonaute (Ago) protein is an essential component of the miRNA-induced silencing complex (miRISC), where it acts as the target recognition component (Ha and Kim 2014; Li and Rana 2014). The target recognition is primarily based on base-pair complementarity between nucleotides $2-8$ of the miRNA (the seed region) and the 3' untranslated region of targeted $\mathrm{mRNA}$, resulting in translational repression, mRNA deadenylation, or mRNA decay (Huntzinger and Izaurralde 2011; Ha and Kim 2014).

The biological functions of miRNAs during teleost gametogenesis and further early embryonic development are poorly understood (Bizuayehu and Babiak 2014). miRNAs are abundant in ovulated oocytes of teleosts (Ma et al. 2012; Ma et al. 2015; Presslauer et al. 2017), and newly formed embryos are transcriptionally quiescent; their development is driven by maternally provided proteins and RNAs (Tadros and Lipshitz 2009; Langley et al. 2014; Lee et al. 2014b). While maternal miRNAs are known to function in model species such Drosophila melanogaster (Marco 2015), Caenorhabditis elegans (McJunkin and Ambros 2017), or mouse (Tang et al. 2007a), to date only miR-202-5p has been confirmed as a maternal miRNA in teleost embryos, but its maternal function remains elusive (Zhang et al. 2017).

Currently, miRNA target prediction is based primarily on computational algorithms, which perform alignments between miRNA sequences and regulatory regions of mRNAs (John et al. 2004; Krek et al. 2005; Friedman et al. 2009). However, these methods often generate many false positive predictions (Ekimler and Sahin 2014) and often do not take into account whether a miRNA and its predicted target are co-expressed (Ritchie et al. 2009). It is therefore important that miRNA/mRNA target interactions are verified experimentally.

Recently, we profiled miRNA transcriptome throughout the gonadal development of zebrafish (Presslauer et al. 2017). miRNA miR-92a-3p was consistently among the most abundant miRNAs in developing and mature gonads of both sexes. 
70 Notably, in ovulated eggs, size variants of miR-92a-3p were dominant, particularly

71 those with one to three additional templated adenine residues (Presslauer et al. 2017).

72 Adenylation of maternal miRNAs in oocytes has been reported in D. melanogaster, sea

73 urchin (Stronglyocentrotus purpuratus), and mouse (Lee et al. 2014a), suggesting miR-

$7492 a-3 p$ may also be stored in zebrafish oocytes with a maternal function in embryos.

75 Previously, we demonstrated that targeted knockdown of miR-92a-3p in mature

76 zebrafish ovaries resulted in a significant reduction of miR-92a-3p in 1-cell embryos,

77 accompanied by a significant increase in the proportion of embryos with arrested

78 development at the 1-cell stage (Presslauer et al. 2016).

79 In the present study, we examine the spatial and temporal expression patterns of

80 miR-92a-3p in zebrafish gonads and early embryonic development. We identify wee1

81 homolog 2 (wee2) as a target for maternal miR-92a-3p, and demonstrate

$82 \mathrm{miRNA} / \mathrm{mRNA}$ co-expression and interactions. 


\section{Results}

\section{miR-92a-3p target prediction}

Twenty-five protein families within the zebrafish cell cycle pathway were identified (Supplementary File S1). Noticeably, the predicted targets were within components involved in DNA biosynthesis, such as the mini-chromosome-maintenance complex and origin recognition complex. In addition, a number of predicted targets are

91 involved in the cyclin dependent kinase (Cdk1) pathway at the G2 / M checkpoint of

92 the cell cycle (Fig. 1). From these predicted Cdk1 pathway targets, wee1 homolog 2

93 (wee2) was selected for target validation assays, based on its confirmed maternal 94 abundance in zebrafish (Aanes et al. 2011).

\section{Spatiotemporal expression in gonads and embryos}

The germ cells in 10-week-old zebrafish testis predominantly consisted of spermatogonia, spermatocytes, and spermatids; whereas in the ovaries, they consisted 100 of stage Ib and II oocytes, with some stage la oocytes located at the periphery. 101 Hybridization with a miR-92a-3p-specific probe identified transcripts in cells of both 102 germline and somatic origin (Fig. 2), with staining particularly strong in primary and 103 vitellogenic oocytes.

104 Both miR-92a-3p and wee2 transcripts were detected in zebrafish embryos. 105 Mature miR-92a-3p transcripts were strongly expressed in zebrafish embryos at all 106 stages examined (Fig. 3 A). At the 1-cell and 256-cell stages, the transcripts were 107 detected ubiquitously within the cells. At the 25 somite stage, miR-92a-3p transcripts 108 were predominantly localized to the eyes and brain, but the signal was detected 109 throughout the body. Expression within the somites was comparatively weaker than in 110 the brain but consistent, while staining was noticeably reduced in the notochord. By 111 comparison, transcripts of wee2 appeared to be strongly expressed at the 1-cell stage.

112 But the signal appeared weaker at the 256-cell stage (Fig. 3 B). At the 25 somite stage, 
113 a faint signal was only detected in the brain and ventral portion of the somites. By

114 comparison, no signal was detected in 1-cell embryos hybridized with negative control 115 probes.

116 Transcripts of miR-92a-3p and wee2 were highly abundant in early stage 117 embryos, with stable levels observed between activated embryos and the oblong 118 stages of development (Fig. 4). At the 25 somite stage, wee2 transcripts were barely 119 detectable; in contrast, miR-92a-3p had a significant increase in transcript abundance.

120 Complete droplet digital PCR results with reference genes are available in 121 Supplementary File S2.

gfp-wee2 reporter expression in embryos

Injections of $200 \mathrm{pg}$ or $800 \mathrm{pg}$ gfp-wee2 resulted in no visible GFP signal (Fig. 5

126 A), similar to non-injected control embryos (Fig. 5 B). Injections of 1600 pg mRNA 127 produced slightly elevated GFP signal within the yolk, while the GFP signal within the 128 body was barely detectable. An increased concentration of $3200 \mathrm{pg}$ gfp-wee 2 resulted 129 in consistent and strong expression of GFP in the embryo (Fig. 5 A). Notably, 130 throughout the experiment, embryos with severe deformities produced intense GFP 131 signal from reduced mRNA concentrations (Fig. 5 C). By comparison, embryos which 132 received injections of chimeric mRNA containing the antisense sequence for the 133 zebrafish wee2 3 'UTR had a strong and ubiquitous GFP signal, which was easily visible 134 from concentrations of 200 pg mRNA onwards, and highly intense at 1000 pg (Fig. 5 D).

\section{miR-92a-3p target site blocker and $g f p-w e e 2$ co-injections}

Co-injection with $g f p-w e e 2$ and either a scrambled control or miR-92a-3p target

139 site blocker (TSB) resulted in varying proportion of GFP expressing embryos and the 140 relative intensity of the GFP signal produced (Fig. 6). Across the three trials, a total of 14136 and 52 embryos were injected with gfp-wee 2 and either the control TSB, or TSB 142 specific to the miR-92a-3p predicted target site, respectively. In total, only six out of 36 
143 embryos (6/36) receiving the control TSB had detectable GFP signal, compared to $14435 / 52$ receiving the miR-92a-3p TSB (Fig. 6 A). In addition, the mean GFP signal 145 intensity in embryos receiving the control TSB was significantly lower than in those 146 receiving the miR-92a-3p TSB (Fig. 6 B; representative embryos in Fig. 6 C).

miR-92a-3p mimic and $g f p-w e e 2$ co-injections miR-92a-3p mimic at a constant dose $(5.0 \mu \mathrm{M})$ demonstrated that the effect of the

152 mimic was overcome only with the high doses of the reporter (Fig. 7 A). Only 2/9 153 embryos injected with 800 pg gfp-wee2 mRNA showed the GFP signal, while no GFP

154 signal was observed in embryos co-injected with the miR-92a-3p mimic (0/5). For the 155 dosage $1600 \mathrm{pg}$, the number of GFP expressing embryos was 19/25 and 1/12, 156 respectively, and for the dosage $3200 \mathrm{pg}$ it was $9 / 10$ and $7 / 8$, respectively, for 157 injections with the reporter and co-injections with the reporter and mimic. While 158 injections of $3200 \mathrm{pg}$ of the reporter were sufficient to overcome the mimic and 159 produce a GFP signal, the GFP intensity was reduced, although not significantly due to 160 the high interspecific variability (Fig. 7 B; representative GFP expressing embryos in Fig. 1617 C).

miR-92a-3p knockdown and rescue

The majority of miR-92a-3p knockdown embryos (23/39) showed delayed 166 development, defined as inability to reach at least the 20-somite stage by 1 day post167 fertilization (dpf); one of these embryos reached only the 1-somite stage. By 168 comparison, all the wildtype embryos reached the 25 -somite stage by $1 \mathrm{dpf}$. A low 169 proportion of morphants were in both rescue and mismatch MO control treatments 170 (4/47 and 2/40, respectively), and no morphants were observed in the miR-92a-3p 171 mimic group (0/39; representative phenotypes given in Fig. $8 \mathrm{~A}$ ). 
172 At $2 \mathrm{dpf}$, embryos from the mismatch $\mathrm{MO}$ control group reached the long pec 173 stage of development, while rescue and miR-92a-3p mimic embryos generally 174 displayed slightly delayed development and appeared closer to the high pec stage of 175 development. The rescue and miR-92a-3p mimic treatments often displayed reduced 176 pigmentation when compared to the MO only treated embryos. Interestingly, the miR177 92a-3p MO-injected embryos, which suffered severe retardation in development at 1 $178 \mathrm{dpf}$, had recovered at $2 \mathrm{dpf}$. They were at the high pec stage of development and exhibited a normal pigmentation pattern, similar to controls (Fig. 8 B).

\section{Discussion}

miR-92a-3p and its predicted target wee2 are abundant maternal factors in zebrafish

Previously we have found miR-92a-3p among the most abundant miRNAs throughout the gonadal development in zebrafish and massively represented in mature oocytes (Presslauer et al. 2017). Also, Vaz et al. (2015) have found this miRNA abundant in mature gonads of zebrafish. The present study demonstrates that the gonadal localization of miR-92a-3p transcripts is in germ cells (Fig. 2) and embryonic localization is within the cell prior to the first cleavage (Fig. 3), providing further evidence of maternal inheritance. Both WISH and ddPCR methods detected high abundance of miR-92a-3p throughout embryogenesis (Figs. 3, 4). The significant increase in miR-92a-3p abundance between the oblong and 25 somite stages (Fig. 4) indicates zygotic transcription as its source in later developmental stages. This pattern differs from the previous reports on zebrafish where miR-92a-3p was suggested to be first expressed during the blastula stage (Li et al. 2011), or where miR-92a-3p gave relatively weak signal at $1 \mathrm{dpf}$, before it greatly increased in expression at 2 and $3 \mathrm{dpf}$

198 (Ning et al. 2013). However, evidence obtained in zebrafish (Presslauer et al. 2016;

199 Presslauer et al. 2017) and rainbow trout (Oncorhynchus mykiss), another teleost fish 200 (Ma et al. 2012), rather clearly suggests that miR-92a-3p is a maternally-inherited 
201 miRNA with functions in the zebrafish embryo prior to the maternal-zygotic transition,

202 and its expression in the later development is of zygotic transcription origin.

203 miR-92a-3p is a member of the conserved miR-17-92 cluster, which functions in 204 regulating mammalian cell cycle, proliferation, and apoptosis (Manni et al. 2009;

205 Mogilyansky and Rigoutsos 2013; Zhou et al. 2015). In human cell lines, miR-92a-3p

206 was determined to function in promoting the cell cycle transition from the $\mathrm{G} 1$ to $\mathrm{S}$

207 phase (Zhou et al. 2015). In zebrafish, miR-92a-3p is most strongly expressed in

208 proliferative organs such as the brain, suggesting its role in regulating cell cycle and

209 proliferation is a conserved function (Ason et al. 2006; Ramachandra et al. 2008).

210 Within the zebrafish cell cycle pathway, we identified a number of predicted targets

211 for miR-92a-3p, including wee2 (Fig. 1, Supplementary File S1). Wee2, a tyrosine

212 kinase, was identified in Xenopus as a Cdk1 inhibitor capable of stopping cell cycle

213 progression (Leise and Mueller 2002; Leise and Mueller 2004). Its role as an oocyte-

214 specific key regulator of meiosis in prophase I and metaphase II through inhibition of

215 CDK1/CDC2 is conserved in mammals, and its deficit leads to fertilization failure in

216 human (Hanna et al. 2010; Sang et al. 2018). The metaphase II arrest is common in

217 vertebrates, including mammals and fish, and its mechanism, termed Cytostatic Factor,

218 is conserved (Madgwick and Jones 2007). However, functions of Wee kinases exert

219 beyond oocyte-specific processes. As demonstrated in Xenopus, the Wee family

220 kinases wee1, wee2, and myt1 have unique spatiotemporal expression patterns during

221 embryogenesis (Leise and Mueller 2002). Notably, while wee1 and myt1 are expressed

222 maternally, Xenopus wee2 is only expressed after the maternal to zygotic transition,

223 where it is predominantly found in locations lacking proliferating cells. However,

224 injections of either wee2 mRNA or protein into 2-cell embryos resulted in delay or

225 arrest of cleavage (Leise and Mueller 2002).

226 In zebrafish, wee2 transcripts are among the most abundant maternal mRNAs

227 during the early stages of embryonic development (Aanes et al. 2011). Our ISH results

228 were consistent with the study by Aanes et al. (2011), in that wee2 was strongly

229 expressed during the cleavage stage, before declining in the blastula stage, and was

230 barely detectable during somitogenesis (Fig. 3 B). The ddPCR results differed in that 
231 expression remained high at the oblong stage before it ceased by the 25 -somite stage

232 (Fig. 4). Both methods indicate that wee2 is a maternally-provided transcript in 233 zebrafish early embryos. In addition, the co-expression of wee2 and miR-92a-3p 234 suggest there is a strong potential for interaction between this miRNA and its potential 235 target.

\section{wee2 is inhibited by endogenous factors}

wee 2 is not among the predicted targets of the miR-430 family (Vejnar and Zdobnov 2012; Agarwal et al. 2015), a major clearance factor of maternal mRNA after maternal-to-zygotic transition (Giraldez et al. 2006). The decline of wee2 transcript level after the maternal-to-zygotic transition suggests that other factors can be involved in its degradation.

244 Injections of a gfp reporter with the wee2 3' UTR antisense sequence at the 245 approximate concentration of 200 pg produced strong and consistent GFP signal (Fig. 5 246 D), similarly to previous studies using GFP reporter assays (Yoshizaki et al. 2005). This 247 was in contrast with the results of injections with reporters having wee 2 3'UTR in the 248 sense orientation, where injections as high as $1600 \mathrm{pg}$ were required for consistent, 249 but faint, GFP signal to be detected (Fig. 5 A). This indicated suppression of wee2 by 250 endogenous factors binding to its 3'UTR. Observations that embryos with severe 251 deformities would produce intense GFP signal from reduced gfp-wee2 concentrations 252 provided further evidence that endogenous factors were acting on the wee2 3'UTR 253 during zebrafish embryogenesis.

254 In zebrafish, miRNAs predicted to target the wee2 3' UTR include: miR-30, miR25596 , miR-101, miR-137, miR-145, miR-148/152, miR-155/2194, miR-156, as well as miR256 25-3p, miR-92a-3p, miR-92b-3p, and miR-363; the four latter ones share the same 257 predicted target site (Agarwal et al. 2015). Among all these miRNAs, miR-92a-3p is by 258 far the most abundant in zebrafish unfertilized eggs, while miR-25-3p is also relatively 259 abundant (Presslauer et al. 2017), suggesting these miRNAs are likely the endogenous 260 factors acting to suppress wee2 translation. 
261 miR-92a-3p suppresses wee2 translation in early development and promotes cell 262 cycle progress

264 The present study demonstrates novel information on miRNA functionality in the 265 cell cycle regulation at the onset of embryogenesis. Several miRNAs are known to be 266 involved in cell cycle regulation (Silva Rodrigues et al. 2018). In mammalian embryonic 267 stem cells, the conserved miR-17-92 cluster supports cellular reprogramming through 268 inhibiting regulatory elements of the $\mathrm{G} 1$ phase and $\mathrm{G} 1 / \mathrm{S}$ checkpoint. At the $\mathrm{G} 2 / \mathrm{M}$ 269 checkpoint, however, the only miRNA with known functionality is miR-195, which 270 suppresses Wee1 transcripts (Mens and Ghanbari 2018).

271 Co-injection of the gfp-wee2 reporter with a target site blocker for the 272 predicted miR-92a-3p binding site at the wee2 3'UTR resulted in strong gfp expression, 273 even at a low concentration of the gfp-wee 2 reporter (Fig. 6). A significant increase in 274 proportion of GFP-expressing embryos, with significantly increased GFP intensity 275 among those embryos, suggested functionality of that binding site in suppression of 276 wee2 by endogenous miRNAs. Co-injection of the $g f p$-wee2 reporter with a miR-92a-3p 277 mimic resulted in a decrease in the number of GFP-expressing embryos, and a 278 decrease in GFP intensity among those embryos (Fig. 7). Together, it demonstrates 279 that miR-92a-3p is functional in the suppressing maternal wee2 during the early 280 development of zebrafish.

281 Previously we observed that the inhibition of maternal miR-92a-3p by injection 282 of a vivo $\mathrm{MO}$ directly into an ovary induced an irreversible developmental arrest of the 283 resulting embryos at the 1-cell stage (Presslauer et al. 2016). In the present study, the 284 delivery of miR-92a-3p MO into 1-cell embryos resulted in a non-lethal phenotype, 285 manifested in a significant proportion of embryos displaying slowed development at 1 $286 \mathrm{dpf}$. The effect was reversible, and the embryos fully recovered by $2 \mathrm{dpf}$ (Fig. 8). These 287 results demonstrate that the timing of the miR-92a-3p-MO application is decisive for 288 the essentiality of unblocking the suppression of the $G 2 / M$ checkpoint. Due to lack of 289 miR-92a-3p, a posttranscriptional suppressor, the concentration of Wee2 in miR-92a-

2903 p knockdown oocytes and further zygotes (Presslauer et al. 2016) was high enough to 
291 block the progression of the first mitotic division; whereas, the miR-92a-3p-MO in the

292 present study was delivered later, into the 1-cell stage embryos. The wee2 transcript

293 level is rather stable throughout the cleavage stages (Fig. 4), a typical feature for

294 maternal transcripts (Tadros and Lipshitz 2009). In the cell cycle regulation context, it

295 means that the concentration of this transcript per nucleus is reduced roughly by half

296 with every cleavage. Thus, the temporal developmental retardation, observed in the

297 present study, could plausibly be associated with diminishing essentiality of Wee2 in

298 the cell cycle suppression, caused by the decreasing concentration of the Wee2 per

299 blastomere. Taken together, the results of the previous (Presslauer et al. 2016) and the

300 current study suggest that miR-92a-3 is essential in promoting the cell cycle

301 progression throughout the first cleavage stages in zebrafish through suppressing

302 translation of CDK1 inhibitors, primarily Wee2.

303 In conclusion, miR-92a-3p is strongly expressed in both gonadal somatic and 304 germline cells in zebrafish. During the early embryonic development, maternal miR$30592 a-3 p$ transcripts are co-expressed with their predicted target wee2, a tyrosine kinase 306 inhibitor of cell cycle progression. Endogenous miRNAs interact with the wee2 3'UTR, 307 and synthetic miR-92a-3p is capable of suppressing gfp-wee2 translation. Knockdown 308 of miR-92a-3p in zebrafish embryos resulted in delayed development during the first 309 day of embryogenesis, while the developmental arrest at the 1-cell stage was observed 310 in the previous study, when the knockdown was performed in mature ovaries. These 311 results suggest that zebrafish maternal miR-92a-3p has essential role in regulating the 312 cell cycle through suppressing CDK1 inhibitors, primarily Wee2. This role is particularly 313 important for the progression through the first cleavage. 


\section{Methods}

\section{Fish}

The zebrafish used in the experiment were from the inbred $A B$ line, which was originally obtained from The Norwegian Zebrafish Platform (zebrafish.no), Norwegian

320 University of Life Sciences (Oslo, Norway), before becoming established at Nord 321 University (Bodø, Norway). The zebrafish were housed in an Aquatic Habitats recirculating system (Pentair, Apopka FL, USA) following standard zebrafish husbandry procedures (Westerfield 2000). Zebrafish nutrition consisted of a daily mix of newly hatched Artemia nauplii (Pentair) and SDS zebrafish specific diet (Special Diet Services, Essex, United Kingdom) following the manufacturers recommended feeding regime.

All experimental procedures described in the present study were performed in accordance with the Norwegian Regulation on Animal Experimentation (The

328 Norwegian Animal Protection Act, No. 73 of 20 December 1974) and were approved by

329 the National Animal Research Authority (Utvalg for fors $\varnothing \mathrm{k}$ med dyr, fors $\emptyset$ ksdyrutvalget,

330 Norway) General License for Fish Maintenance and Breeding (Godkjenning av avdeling 331 for fors $\emptyset \mathrm{ksdyr}$ ) no. 17.

\section{Sampling}

Total RNA was extracted from the ovaries of three mature zebrafish. The fish 336 were first euthanized with 200 mg/L of MS-222 Tricaine; Sigma Aldrich, Oslo, Norway) 337 buffered with equal parts sodium bicarbonate $\left(\mathrm{NaHCO}_{3}\right.$; Sigma Aldrich). After 338 opercular movement had ceased, the fish were decapitated, the gonads were 339 immediately removed, and total RNA was extracted using QIAzol Lysis Reagent 340 (Qiagen, Hilden, Germany) following the manufacturer's instructions. RNA integrity 341 was assessed using $1 \%(\mathrm{w} / \mathrm{v})$ agarose gel electrophoresis and was quantified using a 342 NanoDrop ND-1000 (Thermo Fisher Scientific, Saven \& Werner AS, Kristiansand, 343 Norway). 
344 Ten-week-old zebrafish were sampled for in situ hybridization (ISH). The fish

345 were euthanized as described above; the abdominal cavity was opened, and the 346 specimens were placed directly into pre-chilled Bouin's solution ( $4{ }^{\circ} \mathrm{C}$; Sigma Aldrich).

347 The samples were fixated overnight at $4^{\circ} \mathrm{C}$ before being dehydrated in a gradient series

348 of ethanol washes (from 25 to $100 \%$ ) and embedded in paraffin wax. The samples 349 were systematically sectioned (6 $\mu \mathrm{m}$ thickness) using a rotary microtome (Microm $350 \mathrm{HM} 3555, \mathrm{MICROM}$ International $\mathrm{GmbH}$, Germany) and were mounted on Polysine 351 slides (Thermo Fisher Scientific). Slides were stored at $4{ }^{\circ} \mathrm{C}$ for a maximum of one week 352 before use.

353 Zebrafish embryos were both fixed for whole mount in situ hybridization (WISH), 354 and snap-frozen for total RNA extraction. Embryos were produced by allowing single 355 pairs of adult zebrafish to spawn naturally while under observation to ensure accurate 356 knowledge of the time of fertilization. After embryos were collected, they were 357 individually examined using a stereomicroscope and staged according to previously 358 established guidelines (Kimmel et al. 1995). For the WISH experiment, zebrafish 359 embryos at the 1-cell, 256-cell, and 25 somite stages were collected and placed directly 360 into pre-chilled $4 \% \mathrm{w} / \mathrm{v}$ paraformaldehyde (PFA; $\mathrm{pH} 7.0$ ) and fixated overnight at $4{ }^{\circ} \mathrm{C}$. 361 The following day the embryos were repeatedly washed with a chilled solution of $0.1 \%$ 362 Tween-20 in phosphate buffered saline solution (PBST) before WISH. For total RNA 363 extraction, three additional spawning's were conducted. In each group of embryos, 364 samples were taken at seven developmental stages: activated $(5 \mathrm{~min}$ post365 fertilization), 1-cell, 4-cell, 32-cell, 256-cell, oblong, and 25 somite. For each sampling, 366 pools of ten embryos were collected and placed directly in QIAzol Lysis Reagent.

368 Reverse transcription

370 Complementary DNA (cDNA) was synthesized from total RNA previously 371 extracted from mature zebrafish ovaries and embryos. Reverse transcription was 372 performed using the Quantitect Reverse Transcription Kit (Qiagen) following the 373 manufacturer's protocol. For ovaries and for embryos, $1.0 \mu \mathrm{g}$ and $100 \mathrm{ng}$ total RNA 
374 input was used, respectively. Reverse transcription of small RNAs was performed for all

375 embryonic stages using the miRCURY LNA RT kit (Qiagen) using the recommended 10

376 ng total RNA input.

377

378 In situ hybridization of zebrafish gonads

A locked nucleic acid (LNA) oligonucleotide probe antisense for the mature form of miR-92a-3p was designed and produced by Qiagen. The probe sequence was ACAGGCCGGGACAAGTGCAATA, and the probe was double digoxigenin labeled the $5^{\prime}$ and $3^{\prime}$ ends. As a negative control, a LNA ${ }^{T M}$ Scramble-miR negative control probe was used (GTGTAACACGTCTATACGCCCA).

The ISH procedure was performed in accordance to the miRCURY LNA ${ }^{\text {tm }}$ microRNA ISH Optimization Kit (Qiagen) with some modifications. Melting of paraffin was performed for $1 \mathrm{~h}$ at $60{ }^{\circ} \mathrm{C}$ the day prior to the ISH experiment. Section permeability was improved using $12 \mu \mathrm{g} / \mathrm{mL}$ Proteinase-K (Roche) for 10 minutes at room temperature. LNA probe hybridization was performed with a probe concentration of $40 \mathrm{nM}$ in microRNA ISH buffer (Qiagen). Hybridization occurred at 30 ${ }^{\circ} \mathrm{C}$ below the RNA Tm ${ }^{\circ} \mathrm{C}\left(57^{\circ} \mathrm{C}\right.$ for both miR-92a-3p and the scrambled control) for $1 \mathrm{~h}$.

\section{Whole mount in situ hybridization of zebrafish embryos}

A CRNA probe for zebrafish wee1 homolog 2 (wee2; accession: NM_001037222) which encompassed the majority of the 3' untranslated region (3' UTR). Because zebrafish wee2 has a transcript variant (wee2-204; ENSDART00000151974) with an alternative stop codon, we also amplified an additional region for sequencing. PCR

400 conditions and oligonucleotide sequences are summarized in Supplementary Table S1. 401 PCR products of the expected size were excised from a $1 \% \mathrm{w} / \mathrm{v}$ agarose gel and 402 purified using the QIAquick gel extraction kit according to the manufacturer's protocol 403 (Qiagen). The CDNA fragment was inserted into the PCR4-TOPO vector and 
404 transformed into OneShot chemically competent E. coli cells. After propagation, the

405 vector was purified using the QIAprep ${ }^{\circledR}$ Miniprep kit (Qiagen) and sequenced in both

406 directions using M13 primers with the Big Dye ${ }^{\circledR}$ Terminator 3.1 (Applied Biosystems)

407 sequencing template preparation method. The sequencing reactions were analyzed at

408 the DNA Sekvenseringslab, University of Troms $\varnothing$, Norway. The confirmed zebrafish

409 wee 2 CDNA fragment was amplified from the PCR4-TOPO vector into linear fragments

410 using the M13 primers. No vectors contained the PCR products of the wee2-204

411 transcript variant. The extracted PCR product was then used as a template for

412 digoxigenin labeled cRNA probe transcription with T3 (anti-sense) and T7 (sense) RNA

413 polymerases (Roche, Mannheim, Germany). The cRNA probes were purified using

$414 \mathrm{LiCl} /$ ethanol precipitation and re-suspended in nuclease-free water before storage at $41580^{\circ} \mathrm{C}$.

416 WISH for miR-92a-3p and wee2 was performed as previously described with 417 minor adjustments (Hall et al. 2003). Embryo permeability was improved using 2.5 $418 \mu \mathrm{g} / \mathrm{mL}$ Proteinase-K (Roche) for $10 \mathrm{~min}$ at room temperature. The miR-92a-3p probe 419 hybridization was performed with a probe concentration of $40 \mathrm{nM}$ for $1 \mathrm{~h}$ at $57^{\circ} \mathrm{C}$, 420 whereas wee2 probe hybridization was performed with a concentration of $1.0 \mu \mathrm{g} / \mu \mathrm{L}$ 421 for $48 \mathrm{~h}$ at $70^{\circ} \mathrm{C}$. Bound probe was conjugated to alkaline-phosphatase labelled anti422 DIG antibody (Roche) overnight at a 1:1000 dilution. The colour reactions were 423 performed at room temperature for 1 and $10 \mathrm{~h}$ for miR-92a-3p and wee2, respectively.

425 Quantitative PCR

Transcript quantification was performed using the QX200 ${ }^{\text {TM }}$ Droplet Digital PCR 428 system (Bio-Rad laboratories, Oslo, Norway) according to the manufacturer's 429 guidelines. Custom oligonucleotides were designed for wee2 (Supplementary Table

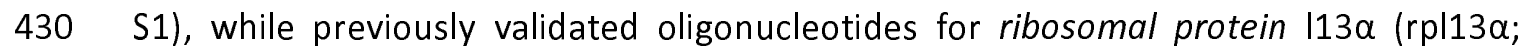
431 accession: NM_001037222) and actin beta 2 (actb2; ENSDART00000055194) were 432 used for comparison (Tang et al. 2007b). For small RNAs, miRCURY LNA ${ }^{\text {tm }}$ miRNA PCR 433 Assays were designed and synthesized by Qiagen for miR-92a-3p as well as miR-19d-3p 
434 (miRBase accession: MIMAT0001785), and miR-192-5p (miRBase accession:

435 MIMAT0001275); the latter two were used for comparison, as they had previously 436 been detected in zebrafish eggs (Presslauer et al. 2017). For both mRNA and small 437 RNA, 20x diluted cDNA inputs were used.

438

miR-92a-3p target validation

Zebrafish miR-92a-3p target validation was performed through a series of 442 microinjection experiments. First, the fragment of wee2 3'UTR, previously amplified 443 for WISH, was excised from the PCR4-TOPO vector using EcoRI digestion (Thermo 444 Fisher Scientific). After gel purification, the fragment was ligated into a pGEM-T-Easy 445 vector (Promega) containing the hrgfp coding region (Yoshizaki et al. 2005). To ensure 446 the proper insert orientation, several colonies were propagated and sequenced as 447 previously described. Plasmids containing both the sense and antisense wee2 3'UTR 448 were linearized using Ndel (Thermo Fisher Scientific) and in vitro transcription was 449 performed using the Message Machine T7 Kit (Thermo Fisher Scientific). The 450 transcribed RNAs were purified using $\mathrm{LiCl}$ precipitation and re-suspended in $20 \mu \mathrm{L}$ 451 nuclease-free water before being aliquoted and stored at $-80^{\circ} \mathrm{C}$. The calculated stock 452 concentrations of $g f p$-wee 2 and $g f p$-wee2-antisense were $3200 \mathrm{ng} / \mu \mathrm{L}$ and $1000 \mathrm{ng} / \mu \mathrm{L}$, 453 respectively (NanoDrop ND-1000).

454 In order to determine the optimal concentration of chimeric RNA for injection, to 455 obtain strong GFP signal, the gfp-wee2 and gfp-wee2-antisense chimeric mRNAs were 456 diluted in freshly made and chilled embryo medium (Westerfield 2000) before injecting 457 into naturally spawned 1-cell zebrafish embryos approximately 30 to $60 \mathrm{~min}$ post 458 fertilization. gfp-wee2 injection concentrations were 3200, 1600, 800, and $200 \mathrm{ng} / \mu \mathrm{L}$ 459 compared to 1000 and $200 \mathrm{ng} / \mathrm{\mu L}$ for the $\mathrm{gfp}$-wee2-antisense control. For all injections, $4601 \mathrm{~nL}$ volume was injected into the yolk directly below the blastodisc using an IM-300 461 microinjector (Narishige, London, UK), resulting in respective dosages of $200 \mathrm{pg}$ to $4623200 \mathrm{pg}$ mRNA. After injection, embryos were transferred to a fresh solution of 463 embryo medium for incubation at $28.5^{\circ} \mathrm{C}$. All observations were performed using an 
464 AxioZoom V.16 microscope (Carl Zeiss, Göttingen, Germany) and Zen Pro (2012; Carl 465 Zeiss) imaging software.

466 The miR-92a-3p target site blocker assays were performed using a miRCURY LNA 467 Power Target Site Blocker (TSB) specific for the miR-92a-3p binding site within the 468 zebrafish wee2 3'UTR. The TSB (5'-ATTATTGCACCCAGTGCC-3'; the miR-92a-3p target 469 site is underlined) was designed and produced by Qiagen, with an additional scrambled 470 TSB to act as a negative control (5'-TAACACGTGTATACGCCCA-3'). Upon receipt, the 471 lyophilized TSBs were re-suspended in nuclease free water at a stock concentration of $47250 \mu \mathrm{M}$ and aliquoted for further use. The TSB assays were optimized for a $1 \mathrm{~nL}$ 473 injection volume consisting of $320 \mathrm{ng} / \mu \mathrm{L}$ gfp-wee 2 and $1.0 \mu \mathrm{M}$ TSB in freshly prepared 474 embryo medium. Microinjections and general observations were made as previously 475 described. The GFP mean signal intensity was measured using the ZEN Measurement 476 Module (Zeiss). For each measurement, contours were traced to encompass the head, 477 brain, and eye region of the embryo; these regions were the most consistent in their 478 GFP expression.

479 mirVana ${ }^{\circledR}$ miRNA mimic for zebrafish miR-92a-3p was designed and produced by 480 Thermo Fisher Scientific. The lyophilized mimic was re-suspended in nuclease-free 481 water to a stock concentration of $100 \mu \mathrm{M}$ and aliquoted for further use. The effect of 482 miR-92a-3p mimic on gfp-wee2 expression was observed by a series of $1.0 \mathrm{~nL}$ 483 injections consisting of $5.0 \mu \mathrm{M}$ mimic and 800,1600 , or $3200 \mathrm{ng} / \mu \mathrm{L}$ gfp-wee2. 484 Observations and measurements were made as previously described.

miR-92a-3p knockdown miR-92a-3p knockdown was performed using a vivo-morpholino (VMO) 489 complementary to the dre-miR-92a-3p guide strand (5'490 TACAGGCCGGGACAAGTGCAATACC-3') designed and produced by Genetools LLC 491 (Philomath, OR, USA) with an additional 5-base mismatch VMO (5'492 TAGACGCCGGACAACTCCAATACC-3'; underlined letters indicate the mismatches) as a 493 negative control. Microinjections and embryo staging were performed as previously 
494 described. Treatments consisted of $5.0 \mu \mathrm{M} \mathrm{VMO}$ and were rescued with an equal

495 concentration of miR-92a-3p mimic. Embryos were examined for phenotypes at 1 and

4962 days post-fertilization.

497

498

miR-92a-3p target prediction

499

500 Targets were predicted for zebrafish miR-92a-3p (miRBase accession:

501 MIMAT0001808) using both TargetScanFish (release 6.2;

502 http://www.targetscan.org/fish_62/) and miRmap (http://mirmap.ezlab.org/) (Vejnar

503 and Zdobnov 2012; Agarwal et al. 2015). Potential targets were filtered using the Kyoto

504 Encyclopedia of Genes and Genomes (KEGG;

505 http://www.genome.jp/kegg/pathway.html) pathway database to select those

506 involved in the zebrafish cell cycle pathway (Kanehisa and Goto 2000).

507

508 Statistical analyses

509

510 Expression values from ddPCR analyses were log transformed and the effect of

511 developmental advancement on transcript abundance was estimated using ANOVA.

512 The Brown-Forsythe test was used to evaluate homogeneity of variances. Tukey's HSD

513 post-hoc test was used to estimate differences in transcript abundance among

514 developmental stages. For GFP-reporter assays, the mean intensity values were tested

515 with F-test for two equal variances before evaluating the effect of the treatment using

516 the two-tailed Student's $t$-test. Percentage data (frequency of GFP expressing embryos

517 and frequency of embryos with delayed development) were arcsin square root

518 transformed. ANOVA was used to analyse differences in development between miR-

519 92a-3p knock-down fish, the mismatch control, miR-92a-3p mimic, and the rescue

520 treatment (morpholino and mimic combination). Multiple comparison analysis was

521 performed using the Tukey-Kramer test. All effects were considered significant at a $p$ -

522 value of 0.05 of less (Zar 1999). 


\section{Acknowledgements}

525

526 We thank the Mørkvedbukta Research Station technicians, in particular Cesilie

527 Amundsen and Katrine Klippenberg, for their assistance in zebrafish husbandry, and

528 Heidi Ludviksen for aid in laboratory operations.

529

$530 \quad$ Funding

531

532 The research was financed by the Research Council of Norway (FishmiR project

533 \# 213825 and InnControl project \#275786), and Nord University.

534

535 Competing financial interests

536 The authors declare no competing financial interests. 
537

538

539

540

541

542

543

544

545

546

547

548

549

550

551

552

553

554

555

556

557

558

559

560

561

562

563

564

565

566

567

568

569

570

571

572

573

574

575

576

577

578

579

580

581

582

\section{References}

Aanes, H., C. L. Winata, C. H. Lin, J. P. Chen, K. G. Srinivasan, S. G. P. Lee, A. Y. M. Lim, H. S. Hajan, P. Collas, G. Bourque et al., (2011) Zebrafish mRNA sequencing deciphers novelties in transcriptome dynamics during maternal to zygotic transition. Genome Res 21, 1328-1338.

Agarwal, V., G. W. Bell, J.-W. Nam and D. P. Bartel, (2015) Predicting effective microRNA target sites in mammalian mRNAs. eLife 4, e05005.

Ason, B., D. K. Darnell, B. Wittbrodt, E. Berezikov, W. P. Kloosterman, J. Wittbrodt, P. B. Antin and R. H. A. Plasterk, (2006) Differences in vertebrate microRNA expression. Proc Natl Acad Sci 103, 14385.

Bartel, D. P., (2009) MicroRNA target recognition and regulatory functions. Cell 136, 215-233.

Bizuayehu, T. T., and I. Babiak, (2014) MicroRNA in teleost fish. Genome Biol Evol 6, 1911-1937.

Ekimler, S., and K. Sahin, (2014) Computational methods for microRNA target prediction. Genes 5, 671-683.

Friedman, R. C., K. K.-H. Farh, C. B. Burge and D. P. Bartel, (2009) Most mammalian mRNAs are conserved targets of microRNAs. Genome Res 19, 92105.

Giraldez, A. J., Y. Mishima, J. Rihel, R. J. Grocock, S. Van Dongen, K. Inoue, A. J. Enright and A. F. Schier, (2006) Zebrafish miR-430 promotes deadenylation and clearance of maternal mRNAs. Science 312, 75.

Ha, M., and V. N. Kim, (2014) Regulation of microRNA biogenesis. Nat Rev Mol Cell Biol 15, 509-524.

Hall, T. E., N. J. Cole and I. A. Johnston, (2003) Temperature and the expression of seven muscle-specific protein genes during embryogenesis in the Atlantic cod (Gadus morhua) L. J Exp Biol 206, 3187-3200.

Hanna, C. B., S. Yao, M. C. Patta, J. T. Jensen and X. Wu, (2010) WEE2 is an oocyte-specific meiosis inhibitor in rhesus macaque monkeys. Biol Reprod 82, 1190-1197.

Huntzinger, E., and E. Izaurralde, (2011) Gene silencing by microRNAs: contributions of translational repression and mRNA decay. Nat Rev Genet 12, 99-110.

John, B., A. J. Enright, A. Aravin, T. Tuschl, C. Sander and D. S. Marks, (2004) Human MicroRNA Targets. PLoS Biol 2, e363.

Kanehisa, M., and S. Goto, (2000) KEGG: kyoto encyclopedia of genes and genomes. Nucleic Acids Res 28, 27-30.

Kimmel, C. B., W. W. Ballard, S. R. Kimmel, B. Ullmann and T. F. Schilling, (1995) Stages of embryonic development of the zebrafish. Dev Dynam 203, 253-310.

Krek, A., D. Grun, M. N. Poy, R. Wolf, L. Rosenberg, E. J. Epstein, P. MacMenamin, I. da Piedade, K. C. Gunsalus, M. Stoffel et al., (2005) Combinatorial microRNA target predictions. Nat Genet 37, 495-500.

Langley, A. R., J. C. Smith, D. L. Stemple and S. A. Harvey, (2014) New insights into the maternal to zygotic transition. Development 141, 3834. 
Lee, M., Y. Choi, K. Kim, H. Jin, J. Lim, T. A. Nguyen, J. Yang, M. Jeong, A. J. Giraldez, H. Yang et al., (2014a) Adenylation of maternally inherited microRNAs by Wispy. Mol Cell 56, 696-707.

Lee, M. T., A. R. Bonneau and A. J. Giraldez, (2014b) Zygotic genome activation during the maternal-to-zygotic transition. Annu Rev Cell Dev Biol 30, 581-613.

Leise, W. F., and P. R. Mueller, (2002) Multiple Cdk1 Inhibitory Kinases Regulate the Cell Cycle during Development. Dev Biol 249, 156-173.

Leise, W. F., and P. R. Mueller, (2004) Inhibition of the cell cycle is required for convergent extension of the paraxial mesoderm during Xenopus neurulation. Development 131, 1703-1715.

Li, N., C. Wei, A. F. Olena and J. G. Patton, (2011) Regulation of endoderm formation and left-right asymmetry by miR-92 during early zebrafish development. Development 138, 1817-1826.

Li, Z., and T. M. Rana, (2014) Therapeutic targeting of microRNAs: current status and future challenges. Nat Rev Drug Discov 13, 622-638.

Ma, H., M. Hostuttler, H. Wei, C. E. Rexroad, III and J. Yao, (2012) Characterization of the rainbow trout egg microRNA transcriptome. PLOS ONE 7, e39649.

Ma, H., G. M. Weber, M. A. Hostuttler, H. Wei, L. Wang and J. Yao, (2015) MicroRNA expression profiles from eggs of different qualities associated with post-ovulatory ageing in rainbow trout (Oncorhynchus mykiss). BMC Genom 16, 201.

Madgwick, S., and K. T. J. C. D. Jones, (2007) How eggs arrest at metaphase II: MPF stabilisation plus APC/C inhibition equals Cytostatic Factor. Cell Div 2, 4.

Manni, I., S. Artuso, S. Careccia, M. G. Rizzo, R. Baserga, G. Piaggio and A. Sacchi, (2009) The microRNA miR-92 increases proliferation of myeloid cells and by targeting p63 modulates the abundance of its isoforms. FASEB J 23, 3957-3966.

Marco, A., (2015) Selection against maternal microRNA target sites in maternal transcripts. G3 (Bethesda) 5, 2199-2207.

McJunkin, K., and V. Ambros, (2017) A microRNA family exerts maternal control on sex determination in C. elegans. Genes Dev 31, 422-437.

Mens, M. M. J., and M. Ghanbari, (2018) Cell cycle regulation of stem cells by microRNAs. Stem Cell Rev 14, 309-322.

Mogilyansky, E., and I. Rigoutsos, (2013) The miR-17/92 cluster: a comprehensive update on its genomics, genetics, functions and increasingly important and numerous roles in health and disease. Cell Death Differ 20, 1603-1614.

Ning, G., X. Liu, M. Dai, A. Meng and Q. Wang, (2013) MicroRNA-92a upholds Bmp signaling by targeting noggin 3 during pharyngeal cartilage formation. Dev Cell 24, 283-295.

Presslauer, C., T. T. Bizuayehu, M. Kopp, J. M. O. Fernandes and I. Babiak, (2017) Dynamics of miRNA repertoire during gonadal development of zebrafish (Danio rerio). Sci Rep 7, 43850.

Presslauer, C., T. T. Bizuayehu, K. Razmi, J. M. O. Fernandes and I. Babiak, (2016) See-Thru-Gonad zebrafish line: developmental and functional validation. Reproduction 152, 507-517.

Ramachandra, R. K., M. Salem, S. Gahr, C. E. Rexroad and J. Yao, (2008) Cloning and characterization of microRNAs from rainbow trout (Oncorhynchus mykiss): 
Their expression during early embryonic development. BMC Developmental Biology 8, 41.

Ritchie, W., S. Flamant and J. E. J. Rasko, (2009) Predicting microRNA targets and functions: traps for the unwary. Nat Meth 6, 397-398.

Sang, Q., B. Li, Y. Kuang, X. Wang, Z. Zhang, B. Chen, L. Wu, Q. Lyu, Y. Fu, Z. Yan et al., (2018) Homozygous mutations in WEE2 cause fertilization failure and female infertility. Am J Hum Genet 102, 649-657.

Silva Rodrigues, D. V., V. V. Silva Monteiro, K. C. Navegantes-Lima, A. L. de Brito Oliveira, S. L. de França Gaspar, L. B. Gonçalves Quadros and M. C. Monteiro, (2018) MicroRNAs in cell cycle progression and proliferation: molecular mechanisms and pathways. Non-Coding RNA Investig 2.

Tadros, W., and H. D. Lipshitz, (2009) The maternal-to-zygotic transition: a play in two acts. Development 136, 3033.

Tang, F., M. Kaneda, D. O'Carroll, P. Hajkova, S. C. Barton, Y. A. Sun, C. Lee, A. Tarakhovsky, K. Lao and M. A. Surani, (2007a) Maternal microRNAs are essential for mouse zygotic development. Genes Dev 21, 644-648.

Tang, R., A. Dodd, D. Lai, W. C. McNabb and D. R. Love, (2007b) Validation of zebrafish (Danio rerio) reference genes for quantitative real-time RT-PCR normalization. Acta Biochimica et Biophysica Sinica 39, 384-390.

Vejnar, C. E., and E. M. Zdobnov, (2012) miRmap: Comprehensive prediction of microRNA target repression strength. Nucleic Acids Res 40, 11673-11683.

Westerfield, M., (2000) The zebrafish book. A guide for the laboratory use of zebrafish (Danio rerio). 4th ed. University of Oregon Press, Eugene.

Yoshizaki, G., Y. Tago, Y. Takeuchi, E. Sawatari, T. Kobayashi and T. Takeuchi, (2005) Green fluorescent protein labeling of primordial germ cells using a nontransgenic method and its application for germ cell transplantation in Salmonidae. Biol Reprod 73, 88-93.

Zar, J. H., (1999) Biostatistical Analysis, 4th edition. Prentice Hall, New Jersey.

Zhang, J., W. Liu, Y. Jin, P. Jia, K. Jia and M. Yi, (2017) MiR-202-5p is a novel germ plasm-specific microRNA in zebrafish. Sci Rep 7, 7055.

Zhou, C., L. Shen, L. Mao, B. Wang, Y. Li and H. Yu, (2015) miR-92a is upregulated in cervical cancer and promotes cell proliferation and invasion by targeting FBXW7. Biochem Biophys Res Commun 458, 63-69. 


\section{Figures}

668 Figure 1. Predicted miR-92a-3p targets within the Cdk1 regulatory pathway. Figure was modified from the KEGG pathway for the zebrafish cell cycle (Kanehisa and Goto 2000). Filled boxes indicate proteins with transcripts predicted as targets for miR-92a-

$6713 p$, whereas empty boxes indicate non-targets.

673 Figure 2. In situ hybridization of miR-92a-3p in 10-week-old zebrafish testes and 674 ovaries compared with hematoxylin and eosin (HE) stained sections and sections 675 hybridized with a scrambled negative control. $\mathrm{Sg}=$ spermatogonia, $\mathrm{SC}=$ 676 spermatocytes, $\mathrm{St}=$ spermatids, $\mathrm{Fc}=$ follicle cells, la = primary growth pre-follicle stage, $677 \mathrm{lb}=$ primary growth follicle stage, $\mathrm{II}=$ cortical alveolus stage. Scalebars represent 100 $678 \mu \mathrm{m}$.

Figure 3. Whole mount in situ hybridization of miR-92a-3p (A) and wee2 (B) at the 1cell, 256-cell, and 25-somite stages. The negative control for miR-92a-3p is a LNA ${ }^{T M}$ Scramble-miR negative control probe, while for wee 2 the antisense sequence of the gene specific probe was used. The scalebar represents $500 \mu \mathrm{m}$.

Figure 4. Droplet digital PCR of miR-92a-3p and wee2 RNAs in zebrafish embryos. Values represent the log2-transformed number of transcript copies per $\mu L$ cDNA input.

687 Error bars are the standard deviation of the log transformed values. Within a 688 transcript, asterisk indicates a significant difference $(p \leq 0.05)$ from all other developmental stages.

Figure 5. GFP-3'UTR chimeric RNA injections into zebrafish embryos. A) Representative images of zebrafish embryos injected with 200,800,1600, or 3200 pg

693 of gfp-wee2 mRNA. GFP signal was detected within the trunks of embryos injected 694 with 1600 or 3200 pg gfp-wee2 mRNA, but not in embryos injected with lower 695 concentrations. B) A non-injected control embryo. C) A representative image for $g f p$ - 
696 wee2-injected embryos showing developmental malformations. D) Representative 697 images of zebrafish embryos injected with 200 or 1000 pg gfp-wee2-antisense mRNA.

698 All observations were made at $1 \mathrm{dpf}$. The scalebar represents $500 \mu \mathrm{m}$.

700 Figure 6. Zebrafish miR-92a-3p target protector assays. Microinjections of $g f p-w e e 2$ 701 chimeric RNA with a target site blocker (TSB) specific for the miR-92a-3p predicted 702 binding site were made to determine miRNA/mRNA interaction. A) The relative 703 proportion of embryos with detectable GFP signal between the control TSB and miR$70492 a-3 p$ TSB treatments. B) Box plots display the distribution of GFP mean intensity 705 values from all embryos in the two treatment groups. Asterisks mark significant 706 differences at $p \leq 0.05$. C) Representative embryos from the non-injected control, gfp707 wee2 and control TSB, and gfp-wee 2 with miR-92a-3p TSB treatments. White contours 708 identify the area of the zebrafish head in which the GFP mean intensity (MI) value was 709 measured (values displayed bottom left). The scalebar represents $500 \mu \mathrm{m}$.

711 Figure 7. Zebrafish miR-92a-3p target validation assays. Microinjections of $g f p-w e e 2$ 712 chimeric RNA with synthetic miR-92a-3p mimic to assess miRNA/mRNA interactions. A) 713 The proportion of embryos with detectable GFP signal after injections with various 714 doses of $g f p$-wee 2 mRNA, and those co-injected with miR-92a-3p mimic $(5.0 \mu \mathrm{M})$. B) 715 Box plots display the distribution of GFP mean intensity values between embryos 716 receiving $3200 \mathrm{pg}$ of $g f p$-wee 2 mRNA, and those also receiving the miR-92a-3p mimic 717 co-injection. Horizontal lines represent the quartiles, with the center horizontal line 718 representing the mean value. C) Representative embryos injected with gfp-wee2 719 mRNA (3200 pg) alone, or co-injected with both gfp-wee2 and miR-92a-3p mimic.

720 White contours identify the area of the zebrafish head in which the GFP mean intensity 721 (MI) value was measured (values displayed bottom left). The scalebar represents 500 $722 \mu \mathrm{m}$.

724 Figure 8. miR-92a-3p knockdown and rescue in zebrafish embryos. A) Representative 725 images display typical development of zebrafish embryos at 1 day post fertilization 
726 after the treatments. Wildtype (non-injected control) embryos are at the 25-somite

727 stage of development, similar to typical embryos which received a scrambled $\mathrm{MO}$, a

728 miR-92a-3p MO and miR-92a-3p mimic co-injection, and a miR-92a-3p mimic only.

729 However, most of miR-92a-3p MO embryos showed retarded development. The chart

730 displays proportion of embryos in each treatment group with the delayed

731 development, as defined by not having reached the 20-somite stage, observed at 1 day

732 post fertilization. Different letters identify groups significantly different from each

733 other between the treatment groups $(p \leq 0.05)$. B) Follow up observations at 2 days

734 post fertilization. Embryos from mimic and $\mathrm{MO}+$ mimic treatments show

735 malpigmentation and slight developmental delay, as compared to control and MO

736 embryos.

737

\section{Supplementary Files}

739

740 Supplementary Table S1. PCR reaction conditions and primer sequences used in the 741 study.

742

743 Supplementary File S1. Predicted cell cycle targets for miR-92a-3p (miRBase

744 accession: MIMAT0001808) using both TargetScanFish and miRmap, and compared

745 to the zebrafish cell cycle KEGG pathway.

746

747 Supplementary File S2. Quantitative PCR of zebrafish maternal miRNAs and mRNAs

748 during early embryogenesis. 


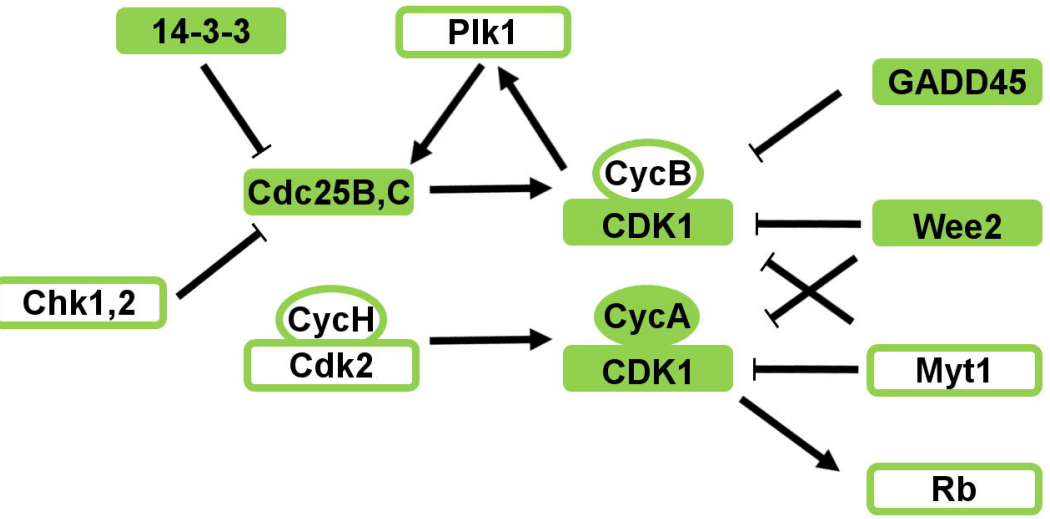

G2 / M Checkpoint 
HE miR-92a-3p

Testis
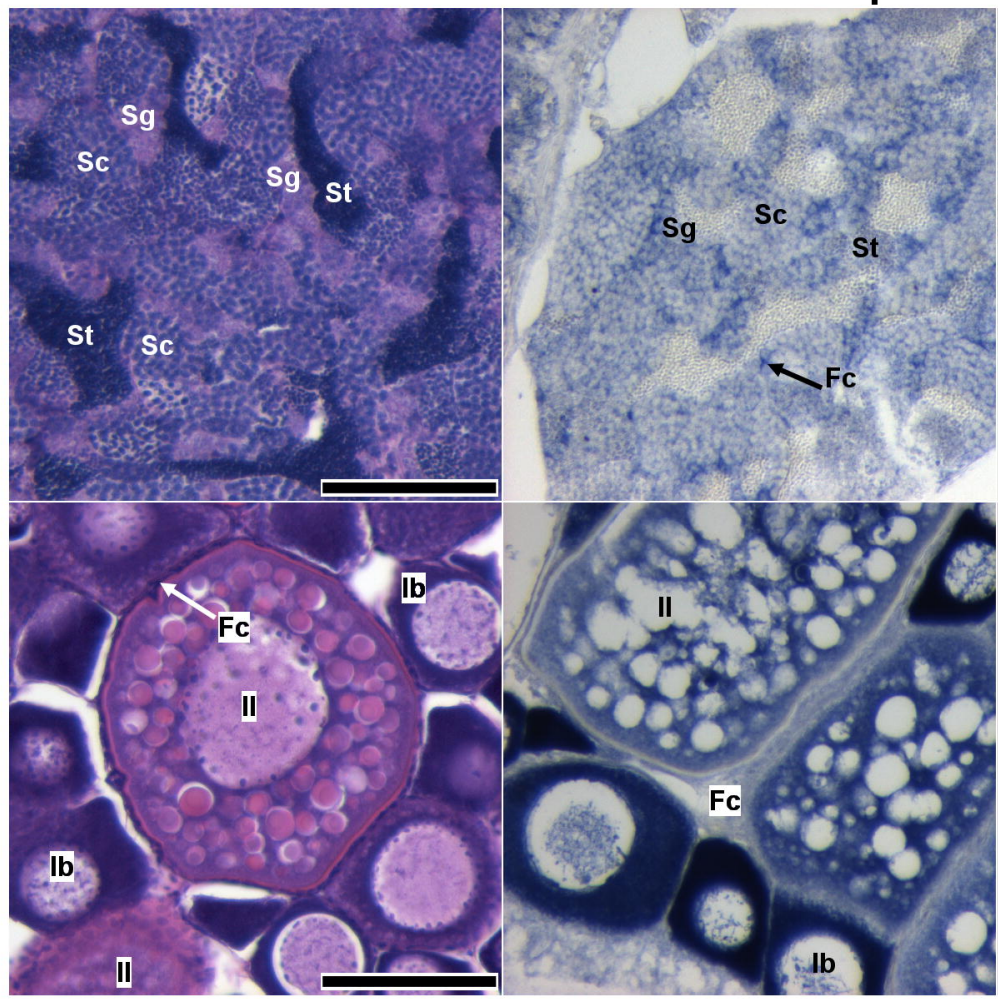

Scrambled control

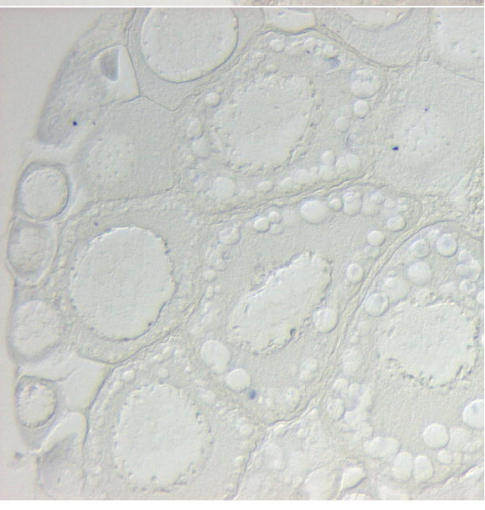


B) wee2

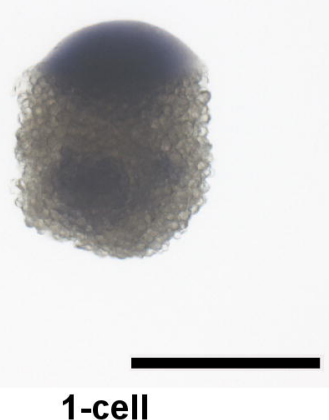

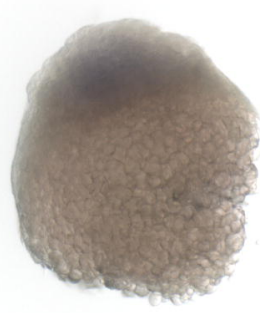

256-cell 25-somite

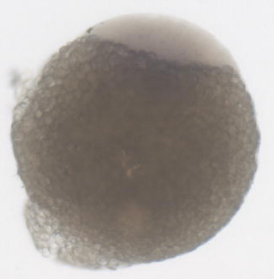

1-cell negative control 


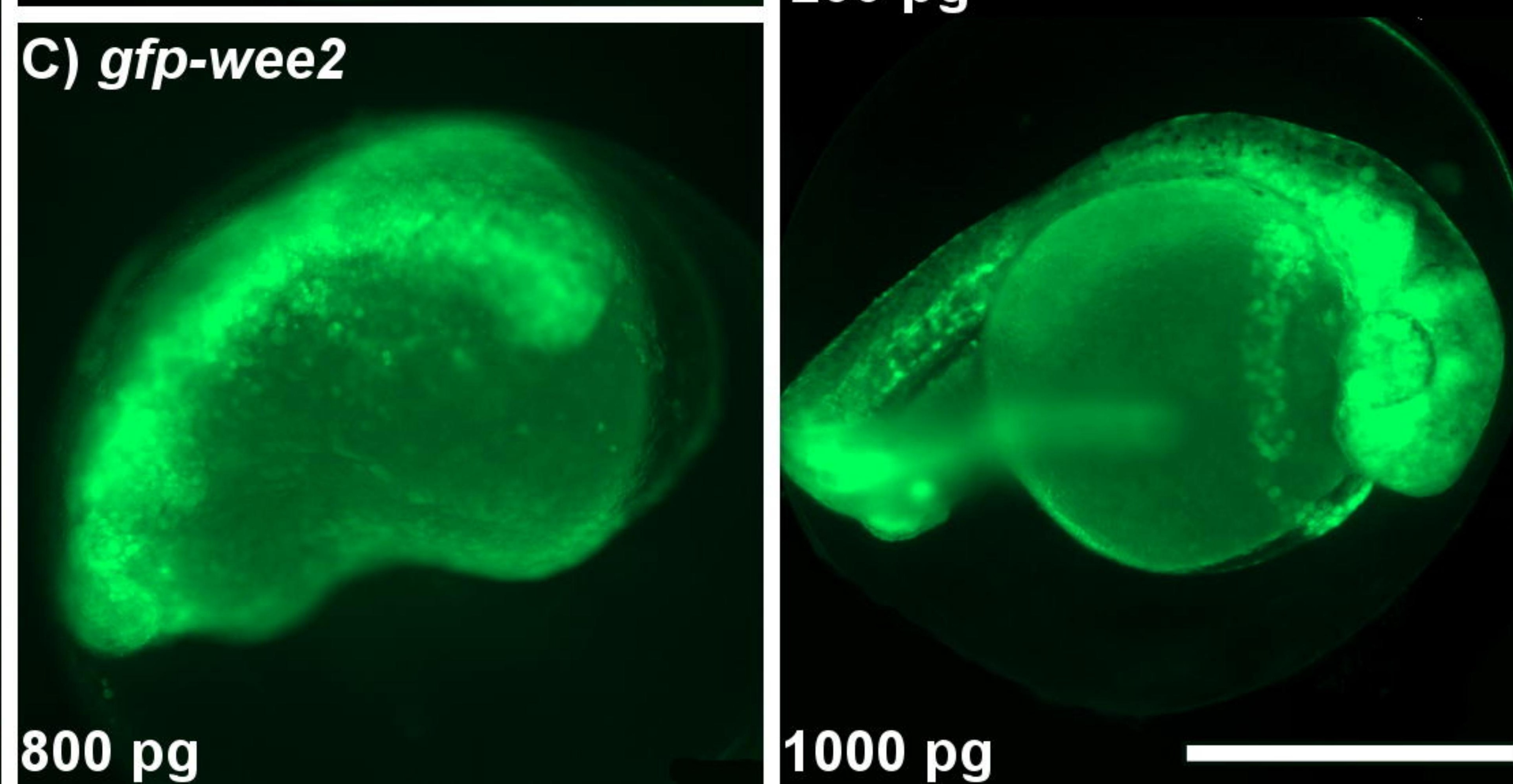


A) 100

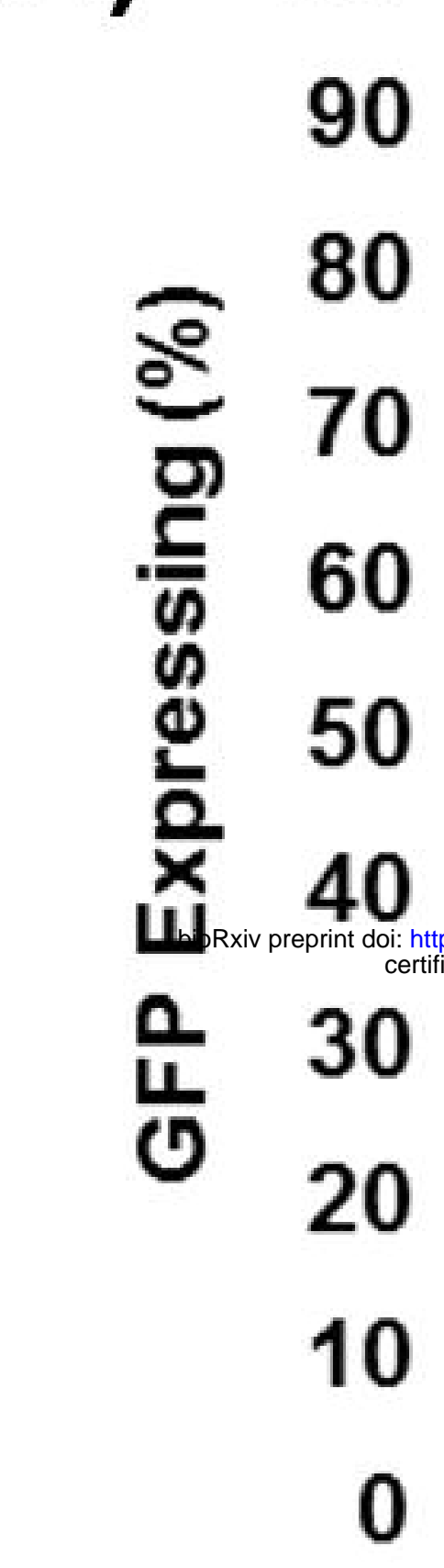

B)
550
要 450
突 350
흐
돈 250
등 150
50

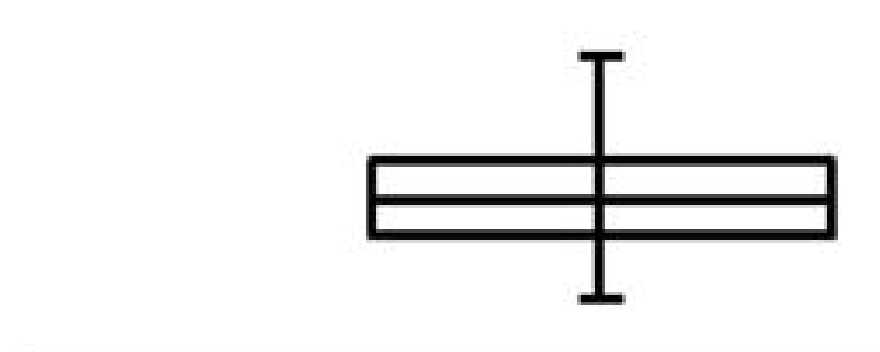

Embryos

Control TSB

- Control TSB

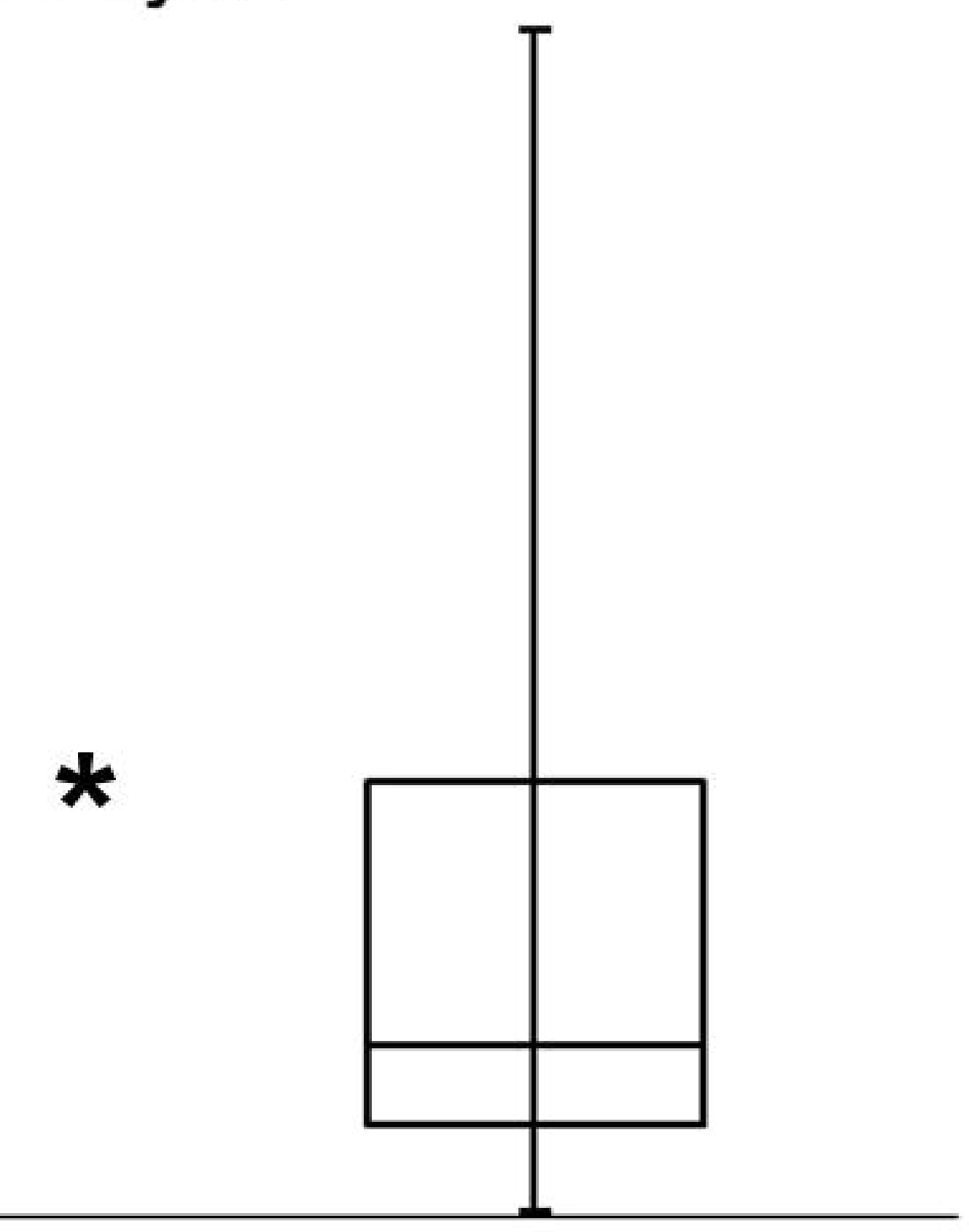

miR-92a-3p TSB

C) Non-Injected Control

gfp-wee2 + control TSB

miR-92a-3p TSB

*

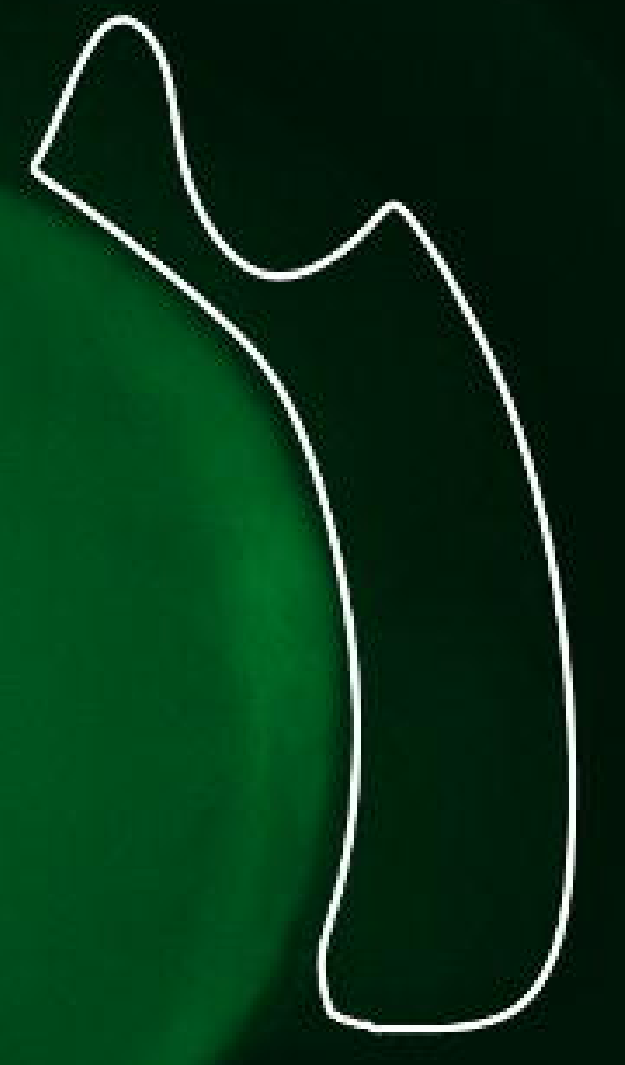

MI: 0

Ml: 101
Ml: 75

gfp-wee2 + miR-92a-3p TSB
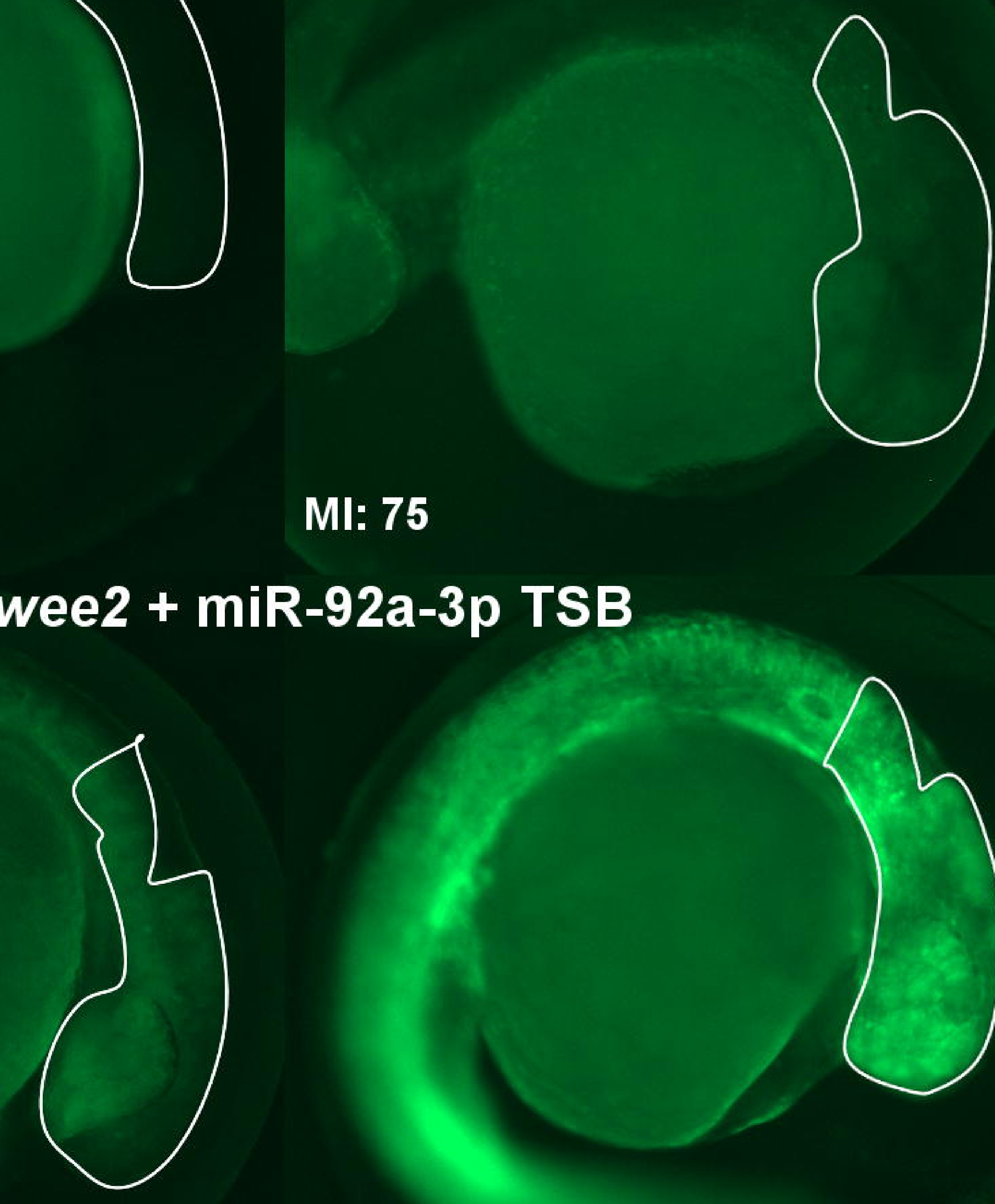

MI: $\mathbf{3 7 4}$ cosuch 
A) $1 \mathrm{dpf}$

\section{Wildtype}

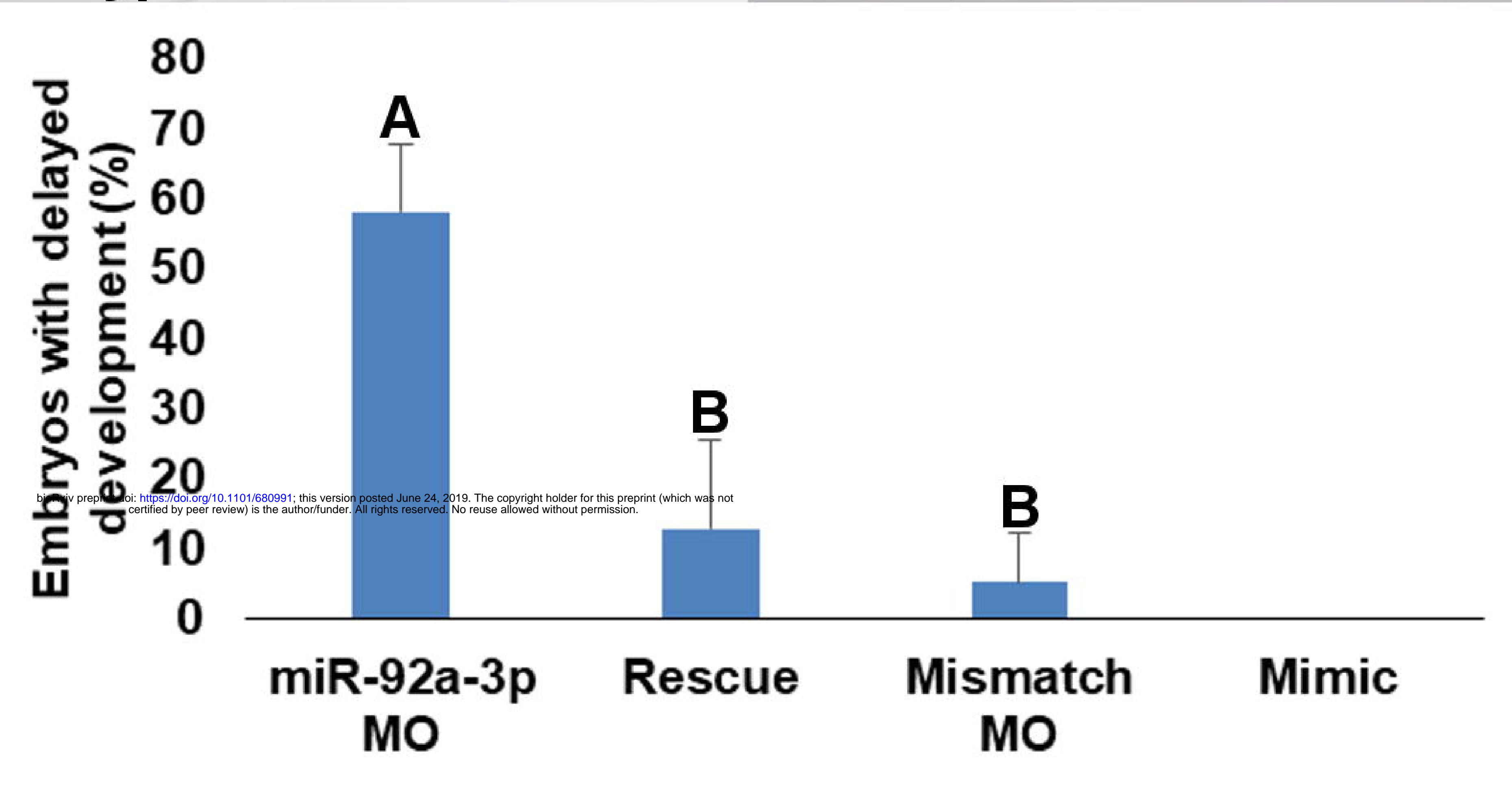

miR-92a-3p MO

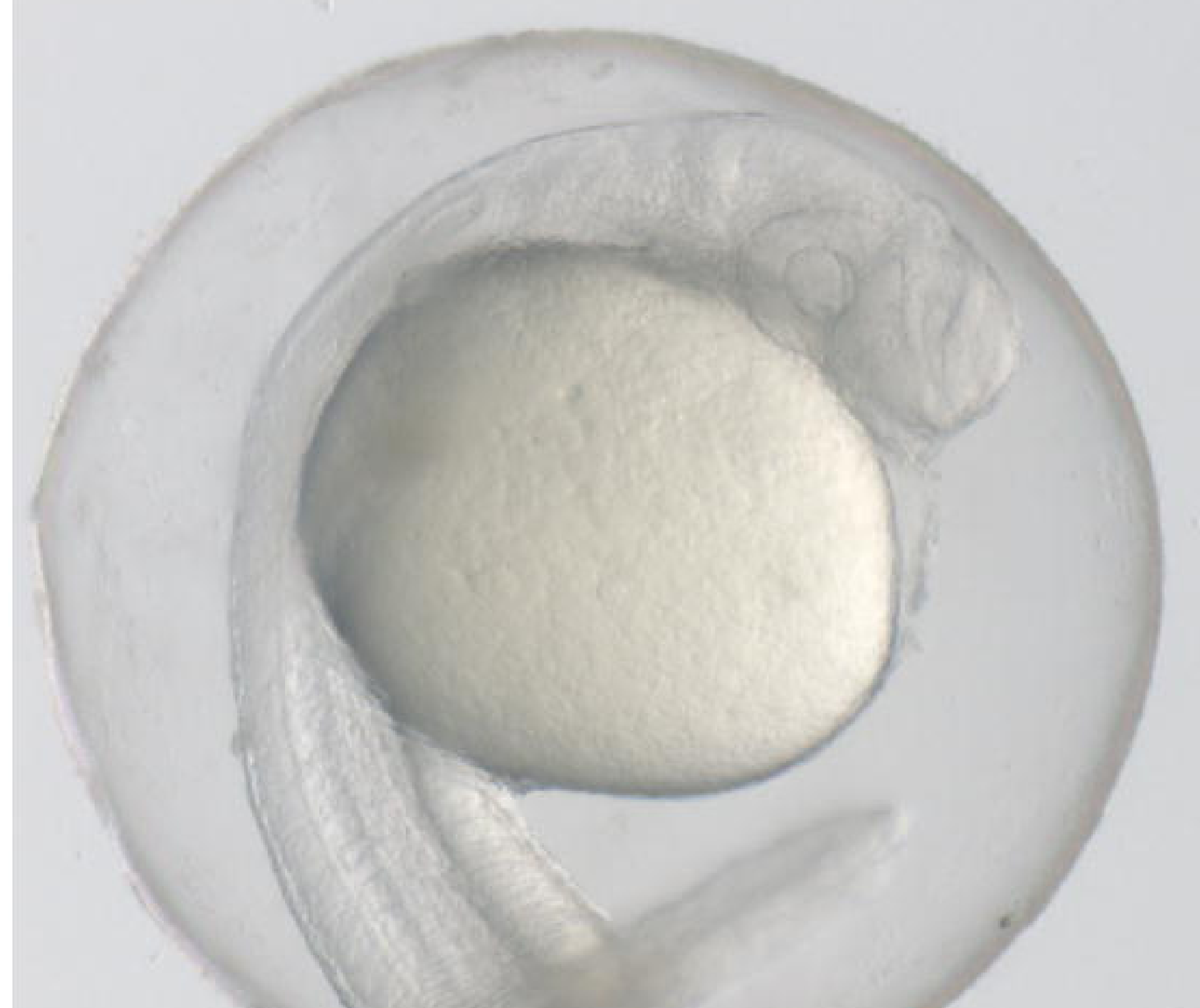

miR-92a-3p MO and mimic
miR-92a-3p MO

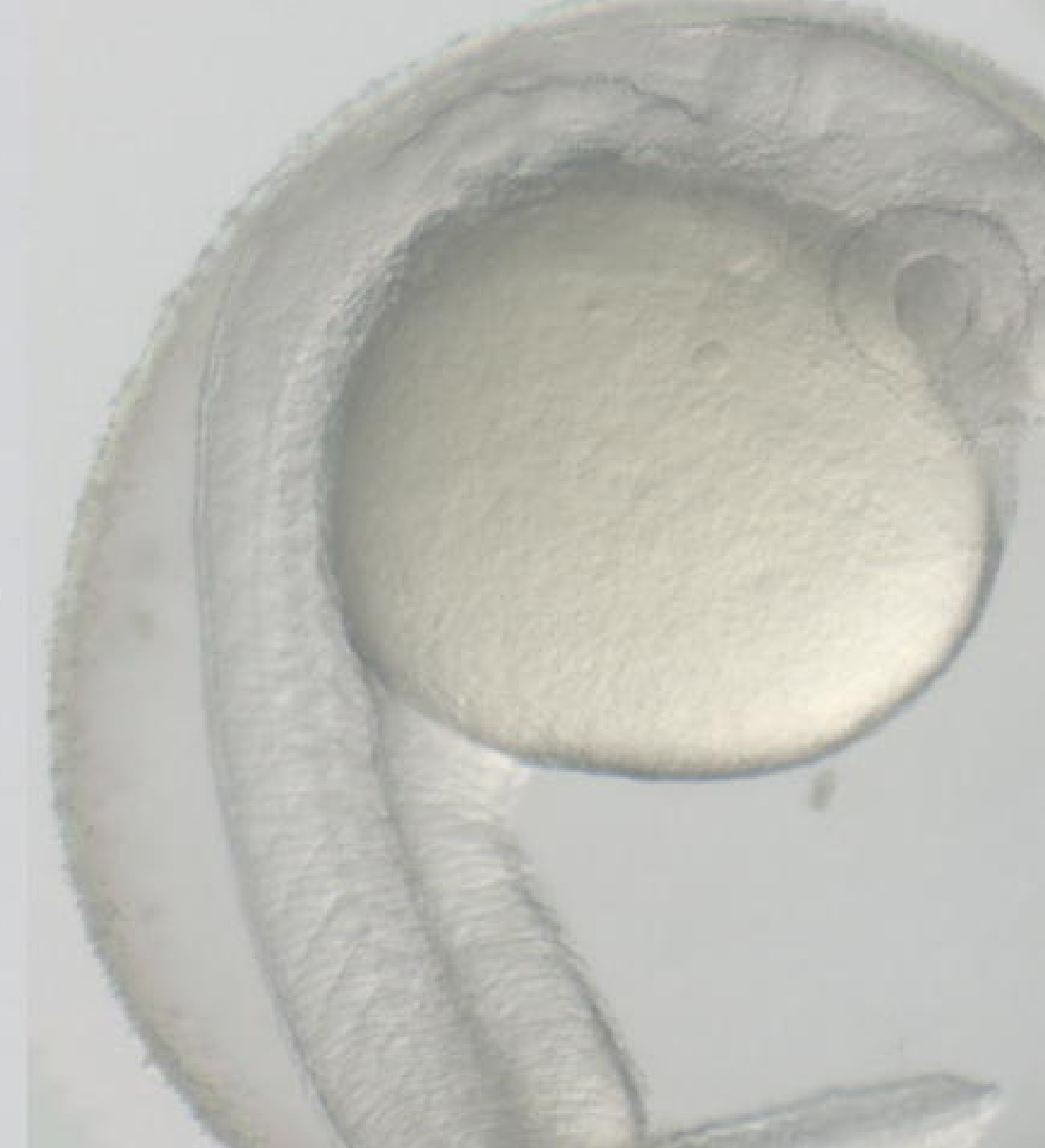

miR-92a-3p mimic

B) $2 \mathrm{dpf}$

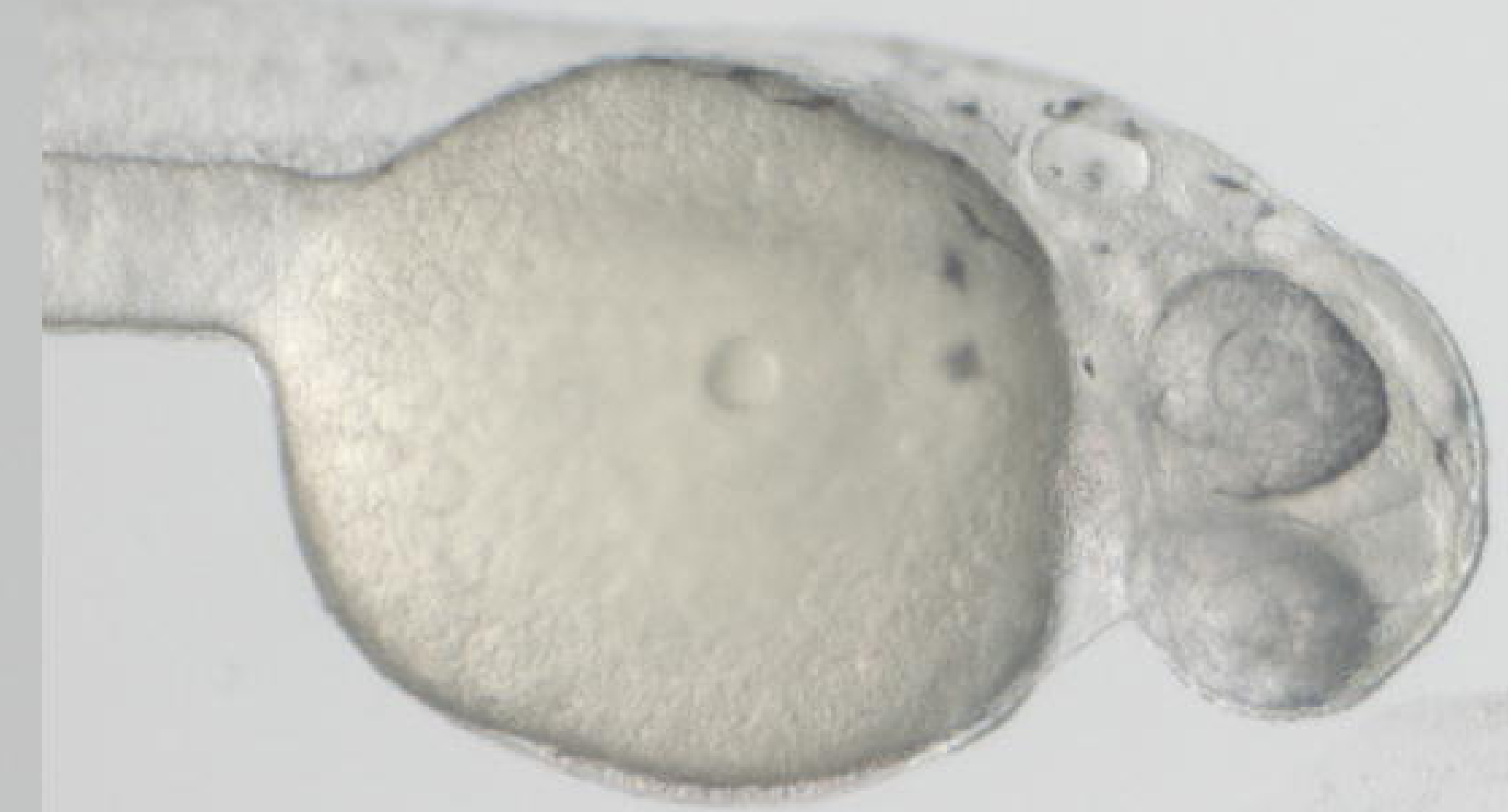

miR-92a-3p MO and mimic miR-92a-3p mimic
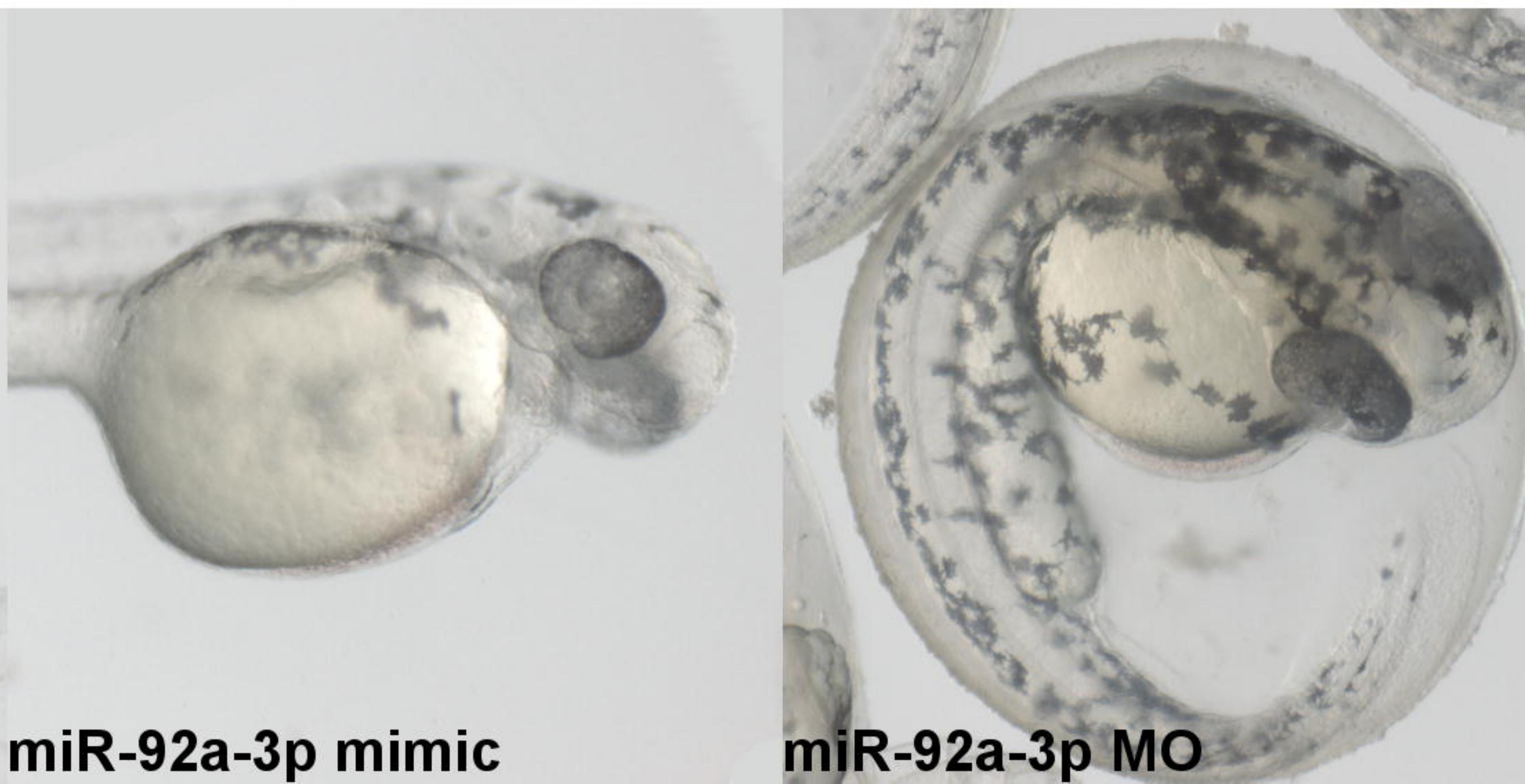